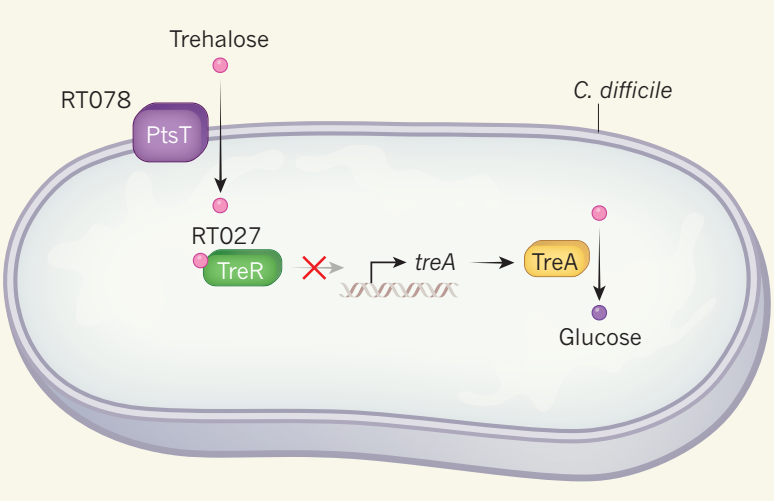

Figure 1 | Increased virulence of the bacterium Clostridium difficile. Two lineages of C. difficile, RT027 and RT078, have become widespread since the early 2000s. Collins et al. ${ }^{6}$ have demonstrated that different mutations have arisen in each strain to improve the microbes' ability to grow on low concentrations of the sugar trehalose, which has been added to foods since 2001. RT078 has acquired four genes, including one that encodes the protein PtsT, which transports trehalose into C. difficile cells. In RT027, mutation of the protein TreR increases the protein's affinity for trehalose, which in turn inhibits TreR's ability to bind to DNA and repress transcription of the gene treA. TreA protein, expressed when TreR is repressed, metabolizes trehalose to glucose and derivatives, enabling cell growth at low trehalose concentrations.

markedly reduced the virulence of this strain in mice. Second, adding trehalose to the diet of mice infected with RT027 increased the animals' risk of death. However, the bacterial load of RT027 was not higher in mice fed trehalose than in those on a trehalose-free diet, indicating that increased risk of death is not simply due to the presence of more bacteria. Rather, the authors found that improved trehalose metabolism enables RT027 to produce higher levels of a $C$. difficile toxin.

Turning to RT078, Collins et al. demonstrated that just one of the four acquired proteins, the trehalose transporter PtsT, was responsible for the strain's increased ability to grow on low levels of trehalose (Fig. 1). The authors showed that PtsT confers a competitive growth advantage over other lineages in the presence of trehalose.

Finally, Collins and colleagues investigated the relevance of their observations in humans. Experimental infection would be difficult in people, so the researchers instead collected fluid from the small intestine of three participants on a normal diet. The fluid contained levels of trehalose sufficient to promote expression of treA in RT027 but not in other strains, supporting the potential for human relevance.

RT027 was first isolated in 1985, from a person infected with $C$. difficile. But this ribotype was not associated with hospital outbreaks, increased death rates or epidemics until the early 2000s. Similarly, RT078 lineages isolated before the $C$. difficile epidemics carry the genetic information for enhanced trehalose metabolism, but were of little consequence to the epidemiology of this disease. Why did these ribotypes suddenly emerge at epidemic levels only 15 years ago?

Collins and colleagues propose a surprising answer. Before 1995, high production costs made trehalose untenable as a food additive. But manufacturing innovations ${ }^{7}$ reduced the cost of trehalose production more than $100-$ fold $^{8}$, and the US Food and Drug Administration and European agencies approved the sugar as a safe food additive in 2000 and 2001, respectively (see go.nature.com/2yewlwk; go.nature.com/2jyltr3). Trehalose is now added to a variety of food products, including pasta, ice cream and minced beef. The authors provide a timeline (see Fig. 6 of the paper) to illustrate how supplementing the food supply with trehalose preceded the $C$. difficile outbreaks caused by RT027 and RT078. They therefore suggest that the addition of trehalose to the food supply might have increased

\section{SUSRUTA MAJUMDAR \& LAKSHMI A. DEVI}

$\mathrm{E}$ ffective pain management is one of the greatest challenges of modern medicine. Opioids such as morphine and fentanyl are the preferred clinical treatments for moderate to severe pain because of their strong analgesic (pain-relieving) effects. But the ongoing epidemic of deaths from respiratory the sugar in the human bowel to levels high enough to enable growth of these ribotypes.

The study's findings raise several avenues for future research. For instance, the connection between trehalose metabolism and toxin production, and how this is linked to increased death rates in people infected with RT027, will require further analysis. Whether trehalose in the human colon, where disease occurs, reaches high enough levels to affect RT027 and RT078 virulence is also unknown. The authors tested fluid from the small intestine, thus bypassing the colon, where the complex complement of gut microbes might break down trehalose.

Despite these concerns, the correlative findings of Collins and colleagues' study are compelling. It is impossible to know all the details of events surrounding the recent $C$. difficile epidemics, but the circumstantial and experimental evidence points to trehalose as an unexpected culprit.

Jimmy D. Ballard is in the Department of Microbiology and Immunology, University of Oklahoma Health Sciences Center, Oklahoma City, Oklahoma 73190, USA.

e-mail:jimmy-ballard@ouhsc.edu

1. Bartlett, J. G. Ann. Intern. Med. 145, 758-764 (2006).

2. McDonald, L. C. et al. N. Engl. J. Med. 353 2433-2441 (2005).

3. He, M. et al. Nature Genet. 45, 109-113 (2013).

4. Hunt, J. J. \& Ballard, J. D. Microbiol. Mol. Biol. Rev. 77, 567-581 (2013).

5. Kuijper, E. J., van Dissel, J. T. \& Wilcox, M. H. Curr. Opin. Infect. Dis. 20, 376-383 (2007).

6. Collins, J. et al. Nature 553, 291-294 (2018).

7. Maruta, K. et al. Biosci. Biotechnol. Biochem. 59, 1829-1834 (1995).

8. Higashiyama, T. Pure Appl. Chem. 74, 1263-1269 (2002).

This article was published online on 3 January 2018.

\title{
Strategy for making safer opioids bolstered
}

Compounds have been made that activate only the G-protein signalling pathway when bound to the $\mu$-opioid receptor - the target of opioid pain relievers. These compounds lack one of the main side effects of currently used opioids.

depression induced by opioid overdoses highlights the need for safer analgesics. Writing in Cell, Schmid et al. ${ }^{1}$ report a series of compounds that provides a much-needed proof of principle of a strategy for making safer opioids.

It is estimated that more than 100 million adults suffer from chronic pain in the United States alone, and that this costs up to US\$635 billion per year in medical treatment 
and lost workforce productivity ${ }^{2}$. The most commonly used drugs for pain management include opioids, non-steroidal anti-inflammatory drugs (NSAIDs) and paracetamol (acetaminophen). However, these treatments can have numerous side effects. For example, NSAIDs can cause cardiovascular complications, gastrointestinal bleeding and renal disease, and acetaminophen is toxic to the liver.

Opioids mainly target the $\mu$-opioid receptor $(\mu \mathrm{OR})$ in neuronal membranes. Activation of the receptor modulates the behaviour of several membrane ion channels along the nociceptive pathways - those neuronal pathways in the nervous system that respond exclusively to painful or potentially painful stimuli - and in central pain-processing centres. But the side effects associated with these drugs include respiratory depression, constipation and addiction. The development of safe, abuse-free opioid analgesics therefore represents a long-standing scientific challenge ${ }^{3}$.

Several strategies have been used to try to develop analgesics that activate the $\mu \mathrm{OR}$ but which are free from adverse side effects. These include the development of partial activators of the $\mu \mathrm{OR}$ (ref. 4); of compounds that activate the $\mu \mathrm{OR}$ but block other opioidreceptor subtypes ${ }^{5}$; and of compounds that are restricted to the peripheral, rather than the central, nervous system $^{6}$. Other approaches have involved molecules that bind to the $\mu \mathrm{OR}$ only in acidic environments ${ }^{7}$ (which are often associated with damaged tissue), and

a

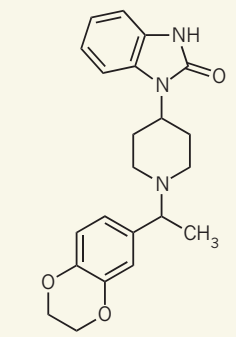

SR-11501

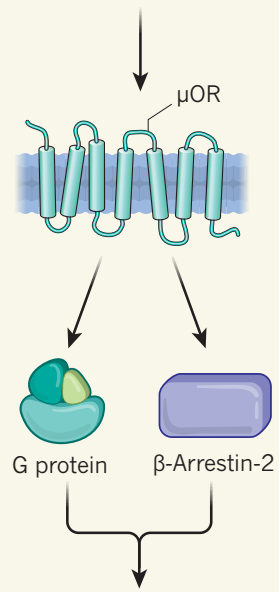

Painkilling effect

Respiratory depression b

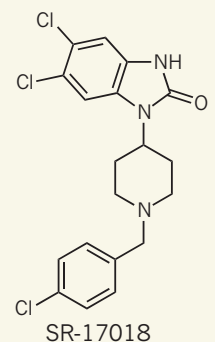

$\downarrow$

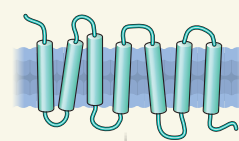

Painkilling effect No respiratory depression
Figure 1 | Signalling preferences correlate with the physiological effects of opioids. Schmid et al. ${ }^{1}$ prepared compounds that bind to and activate the $\mu$-opioid receptor $(\mu \mathrm{OR})$ - the biological target of opioid painkillers. a, $\mu \mathrm{OR}$ activation by some compounds, such as SR-11501, triggers signalling through two pathways: the G-protein pathway and the $\beta$-arrestin- 2 pathway. Such compounds are pain relievers in mice, but cause respiratory depression as a side effect. b, By contrast, compounds such as SR-17018, which trigger only G-protein-mediated signalling, retain their pain-relieving activity but do not cause respiratory depression. compounds that target opioid recep-

tors formed from more than one subtype ${ }^{8}$. A more-recent strategy has been to make allosteric modulators of the $\mu \mathrm{OR}-\mathrm{com}$ pounds that activate it by binding to a region on the receptor other than its active site ${ }^{9}$. Each of these approaches has enhanced our understanding of the $\mu \mathrm{OR}$ system, but has not led to the identification of a safe analgesic.

In the past few years, it has become increasingly evident that different ligand molecules can activate receptors in different ways ${ }^{10}$. In the case of G-protein-coupled receptors (the family of receptors to which the $\mu$ OR belongs), some ligands selectively activate a signalling pathway that involves the eponymous $G$ protein, whereas others activate signalling through a protein called $\beta$-arrestin- 2 . This selectivity is called biased agonism.

Morphine has no bias for the G-protein or $\beta$-arrestin- 2 signalling pathways. In 1999, a study ${ }^{11}$ showed that the analgesic effect of morphine is enhanced in mice that lack $\beta$-arrestin- 2 compared to the effect in wildtype mice, and that several of the drug's side effects were reduced. Other studies have since shown similar effects of morphine in mice in which the expression of $\beta$-arrestin- 2 has been downregulated or inhibited ${ }^{12,13}$. These findings support the idea ${ }^{14}$ that opioid agonists with a strong bias towards G-protein-mediated signalling will retain their analgesic properties, but produce fewer side effects than unbiased opioids. Several laboratories have since identified G-protein-biased $\mu \mathrm{OR}$ agonists ${ }^{4,15-19}$, many of which clearly separate analgesia from adverse side effects. One of these compounds, known as TRV130, is currently in phase III clinical trials ${ }^{16}$. But how well the G-protein bias of these compounds correlates with analgesic efficacy and the reduction of unwanted side effects is not clear.

Schmid et al. tackle the issue of biased opioid signalling head on, and suggest a way to develop analgesic opioids that do not cause respiratory depression. The authors used a previously unreported, selective $\mu \mathrm{OR}$ agonist as a starting point, and synthesized analogues of the compound that contained modifications in two regions of the molecule. They then assessed the analogues in vitro for signalling bias, and in vivo for analgesic efficacy and effects on respiratory depression in mice. All of the compounds were found to be $\mu \mathrm{OR}$ agonists and produced $\mu \mathrm{OR}$-dependent analgesia.

The authors observed a robust relationship between in vitro G-protein bias and in vivo analgesia and respiratory depression: compounds that exhibited higher G-protein bias were stronger pain relievers and caused less respiratory depression. For example, they observed that fentanyl and one of their new compounds (SR-11501; Fig. 1a) exhibit arrestin bias and have a narrow, threefold therapeutic window - that is, the dose of the compounds at which analgesia occurs is approximately three times lower than the dose at which respiratory depression occurs. By contrast, another compound (SR17018; Fig. 1b) has a robust G-protein bias and a more than 25 -fold therapeutic window.

Schmid and colleagues report that SR-17018 does not activate the $\beta$-arrestin- 2 signalling pathway in vitro even at high concentrations, and does not block arrestin recruitment by classic $\mu \mathrm{OR}$ agonists. These results suggest that SR-17018 stabilizes a conformation of $\mu \mathrm{OR}$ that has no affinity for $\beta$-arrestin-2, supporting the idea that the pharmacological effects of SR-17018 are attributable solely to its G-protein bias. This distinguishes SR-17018 from previously reported $\mu \mathrm{OR}$ ligands.

These results are exciting, but it remains to be seen whether G-protein-biased compounds could be developed as non-addictive opioid analgesics. A few promising drug candidates have been identified that might help to answer this question - for example, in mice, the preclinical candidates PZM21 (ref. 17) and mitragynine pseudoindoxyl ${ }^{19}$ exhibit some G-protein bias and clearly separate $\mu \mathrm{OR}$-mediated analgesia from adverse side effects, including respiratory depression, constipation and capacity for abuse. However, a study in rodents indicates that TRV130 retains the potential for abuse ${ }^{20}$.

Nevertheless, compounds that have similar properties to those identified by Schmid and colleagues are strong candidates for the development of truly safe analgesics. Rational approaches to the design of such a drug will require the identification of the amino-acid residues in the $\mu$ OR's binding pocket that are responsible for biased agonism. A longterm goal must therefore be to generate crystals of the receptor in its G-protein-biased and arrestin-biased conformations, so that 
the structures can be solved using X-ray crystallography. The compounds identified by Schmid et al. should also inform our understanding of signalling through G-proteincoupled receptors in general. Given that such receptors are implicated in many diseases, this could pave the way for the development of numerous drugs that have minimal side effects.

Susruta Majumdar is in the Department of Neurology, Memorial Sloan Kettering Cancer Center, New York, New York 10065, USA. Lakshmi A. Devi is in the Department of Pharmacological Sciences, Icahn School of Medicine at Mount Sinai, New York,

New York 10029, USA.

e-mails:majumdas@mskcc.org;

lakshmi.devi@mssm.edu

1. Schmid, C. L. et al. Cell 171, 1165-1175 (2017).

2. Institute of Medicine. Relieving Pain in America (Natl Acad. Press, 2011).

3. Pasternak, G. W. \& Pan, Y.-X. Pharmacol. Rev. 65, 1257-1317 (2013).

4. Grinnell, S. G. et al. Synapse 70, 395-407 (2016).

5. Ananthan, S. AAPS J. 8, E118-E125 (2006).

6. Eans, S. O. et al. J. Med. Chem. 58, 4905-4917 (2015).

7. Spahn, V. etal. Science 355, 966-969 (2017).

8. Fujita, W., Gomes, I. \& Devi, L. A. Br. J. Pharmacol. 172, 375-387 (2015).

9. Burford, N. T. et al. Proc. Natl Acad. Sci. USA 110,
10830-10835 (2013)

10. Rankovic, Z., Brust, T. F. \& Bohn, L. M. Bioorg. Med. Chem. Lett. 26, 241-250 (2016).

11.Bohn, L. M. et al. Science 286, 2495-2498 (1999).

12.Bu, H., Liu, X., Tian, X., Yang, H. \& Gao, F. Int. J. Neurosci. 125, 56-65 (2015).

13.Li, Y. et al. Int. J. Mol. Sci. 10, 954-963 (2009).

14. Raehal, K. M., Walker, J. K. \& Bohn, L. M. J. Pharmacol. Exp. Ther. 314, 1195-1201 (2005).

15. Harding, W. W. et al. J. Med. Chem. 48, 4765-4771 (2005).

16.DeWire, S. M. et al. J. Pharmacol. Exp. Ther. $\mathbf{3 4 4}$ 708-717 (2013).

17. Manglik, A. etal. Nature 537, 185-190 (2016).

18.Kruegel, A. C. et al. J. Am. Chem. Soc. 138, 6754-6764 (2016).

19.Váradi, A. et al. J. Med. Chem. 59, 8381-8397 (2016).

20.Altarifi, A. A. et al. J. Psychopharmacol. 31, 730-739 (2017).

\section{Homing in on a key factor of climate change}

\section{The sensitivity of Earth's climate to atmospheric carbon dioxide levels is a big unknown in predicting future global warming. A compelling analysis suggests that we can rule out high estimates of this sensitivity. SEE LETTER P.319}

uncertain. It has also become a focus for those who doubt the robustness of climate science, who use it to suggest that the field as a whole is intrinsically unreliable. Despite the huge progress in our understanding of climate science over the past 40 years, the Intergovernmental

Panel on Climate Change (IPCC) concluded $^{1}$ in 2013 that there is a $66 \%$ likelihood of ECS being between $1.5^{\circ} \mathrm{C}$ and $4.5^{\circ} \mathrm{C}$ (Fig. 1). This is little different from the range first postulated ${ }^{6}$ by the meteorologist Jule Charney and colleagues in 1979.

Cox and co-workers' estimate is exciting because it develops an underexplored line of evidence: the natural variability of global temperature. The authors also provide the first convincing evidence that we are not living in a world in which ECS is greater than the range of values thought likely by the IPCC. This is important, because estimates of ECS based on the historical temperature record have largely been unable to exclude high values that would invariably result in world-devastating warming of $4^{\circ} \mathrm{C}$ or more by 2100 .

Past research that seemingly constrained the top end of ECS estimates to lower values often excluded major uncertainties, or worked from a previous estimate of ECS that was skewed towards low values. The published ranges therefore depended on the researchers' assumptions about ECS, rather than the evidence. By contrast, Cox et al. started from climate-model values that are at the upper end of the IPCC range, and used evidence to effectively rule out catastrophically high values: they estimate that there is a $66 \%$ likelihood of ECS being between $2.2^{\circ} \mathrm{C}$ and $3.4^{\circ} \mathrm{C}$, with less than a $1 \%$ chance of it being greater than $4.5^{\circ} \mathrm{C}$ (Fig. 1).

The idea underpinning this work

Figure 1 | Estimates of equilibrium climate sensitivity (ECS). ECS quantifies the increase in Earth's average surface temperature that would occur if atmospheric carbon dioxide levels were doubled and the climate system was allowed to reach an equilibrium state. Estimates of ECS vary depending on the evidence used (such as records of Earth's energy budget ${ }^{9}$ and analyses ${ }^{4}$ of present climate conditions produced by models). The estimate ${ }^{1}$ from the Intergovernmental Panel on Climate Change (IPCC) published in 2013 is based on several lines of evidence. Cox et al. ${ }^{5}$ now report estimates based on an analysis of surfacetemperature variation predicted by climate models. Their analysis rules out high estimates of ECS. Bars depict ranges for which there is a $66 \%$ likelihood of the value being correct; for the top two bars, these ranges have been inferred from the data in references 4 and 9 . Best estimates of ECS for each range, if available, are indicated by a blue line. is so enviably simple that it will make climate scientists ask, "Why didn't I think of that?" The authors examined the variability of surface temperature in terms of its variance and autocorrelation - the 'memory' of a previous year's surface temperature that is retained in measurements taken the following year. They then developed a theory-derived metric of surfacetemperature variability and evaluated this metric in historical simulations 


\section{CORRECTION}

The News \& Views 'Strategy for making safer opioids bolstered' by Susruta Majumdar and Lakshmi A. Devi (Nature 553, 286-288; 2018) incorrectly stated that more than 100,000 adults suffer from chronic pain in the United States. The correct figure is more than 100 million adults. 